\title{
Historia de la Neurocirugía en el Hospital Provincial de Guipúzcoa
}

\author{
E. Úrculo-Bareño
}

Servicio de Neurocirugía. Hospital Donostia. San Sebastian.

\section{Resumen}

El objetivo de esta reseña histórica es dar a conocer la evolución de la asistencia neuroquirúrgica en el Hospital Provincial de Guipúzcoa desde su creación en 1960 hasta su desaparición en 2000, tras la fusión de los Servicios y Hospitales en un único centro llamado inicialmente Complejo Hospitalario Donostia, actualmente bautizado con el nombre de Hospital Donostia.

PALABRAS CLAVE: Hospital Provincial. Hospital de Guipúzcoa. Historia. Neurocirugía.

History of Neurosurgery at the Provincial Hospital of Guipuzcoa

\section{Summary}

The purpose of this historical review is to make known the gradual development of neurosurgery at the Provincial Hospital of Guipuzcoa since its creation in 1960 until its disappearance in 2000, after the union of the Hospitals and Services in one single institution called firstly Donostia Hospital Complex, actually known by the name of Donostia Hospital.

KEY WORDS: Provincial Hospital. Guipuzcoa Hospital. History. Neurosurgery.

\section{Introducción}

La neurocirugía en Guipúzcoa y en particular en el Hospital Provincial, viene de la mano de D. Mariano Arrazola Silio, mi maestro, (a quien se le conoce popularmente con el nombre de "Don Mariano" y a quien, privada y cariñosamente sigo llamándole "jefe"). Don Mariano Arrazola nació en Madrid el 30 de Noviembre de 1922. Después de cursar sus estudios de Medicina y Cirugía en la Facultad de San Carlos en Madrid, se decanta por la neurocirugía, entrando

Recibido: 8-10-08. Aceptado: 12-12-08 a formar parte del grupo liderado por el Dr. Obrador. Allí hace relación con el grupo de pioneros y aventureros de la incipiente neurocirugía madrileña, haciendo gran amistad, entre otros, con el Dr. Anastasio, de quienes guardo para mi varias anécdotas contadas por boca de mi maestro, alguna de ellas descrita insuperablemente por José María Izquierdo en su excelente libro "El doctor Obrador en la Medicina de su tiempo". Después de sus primeros años de formación neuroquirúrgica en Madrid y en una de las reuniones organizadas por D. Sixto Obrador, contacta en 1949 con el Dr. Henk Verbiest (jefe del departamento de neurocirugía de la Universidad de Utrecht, Holanda, con reconocido prestigio internacional, entre otras cosas por hacer la primera descripción de la estenosis de canal lumbar) y, lo que iba a ser una estancia de tres meses en Utrecht, se convierte en una formación de tres años, con boda incluida, contrayendo matrimonio con la mejor instrumentista del Prof. Verbiest: Marta Schlamilch ("Toetie"). A sus 30 años y de regreso a España, deciden trasladarse a la ciudad de San Sebastián, porque es una ciudad de categoría y está a pocos minutos de Francia y de su pasión: la montaña. Se establece en San Sebastián en el año 1953, un año después que D. Pedro Albert lo hiciera en Sevilla y que a D. Sixto Obrador le designaran Jefe del Servicio de Neurocirugía del Hospital de la Princesa ${ }^{8}$. Entra a formar parte de la Sociedad Luso-Española de Neurocirugía (SLEN) junto con Anastasio, Jacas, Ley Palomeque y Lobo Antunes en la primera Reunión Conjunta con la "British Society of Neurological Surgeons" celebrada en Madrid en $1951^{17}$.

Aunque existía en la ciudad de San Sebastián antecedentes de intervenciones quirúrgicas craneales, practicadas las más de las veces por cirujanos generales con mayor o menor éxito, el Dr. Arrazola introduce lo que podemos llamar la moderna Neurocirugía. La sociedad en general desconocía lo que era un neurocirujano y menos aún la patología que trataba, peor aun: pensaban que la cirugía intracraneal era el último eslabón para el paciente, ya desahuciado de antemano (aun hoy en día algunos colegas nos recuerdan en plan de broma el dicho popular "coco que se abre, coco que se seca"). Como apuntaba el Dr. Lamas ${ }^{13}$ : "la década de los cincuenta fue clave para comprender el 
proceso que termina por convertir una "extraña" y hasta entonces mal comprendida especialidad médico-quirúrgica, en algo habitual y rutinario en el quehacer hospitalario de nuestro país". Para comprender las dificultades encontradas por estos pioneros, debemos recordar que, entre otras cosas, la especialidad de Neurocirugía es reconocida como tal en nuestro país en la ley de especialidades del 20 de Junio de $1955^{1}$ y su evolución, aunque con cierto retraso, similar a la de otros países $5,7,10,12,24$. En 1956 publicó junto a Obrador, Albert, Anastasio, Boixadós, Sánchez Juan y Vázquez Añón una excelente monografía sobre lesiones degenerativas y malformativas del estuche cráneo-vertebral ${ }^{4}$. Previamente, D. Ricardo Bueno Ituarte, buen neurólogo clínico con posterior afición quirúrgica que se inició en Alemania con Foerster, realiza en San Sebastián intervenciones neuroquirúrgicas, llegando a publicar en la Revista "Guipúzcoa Médica" (1933) dos trabajos titulados: un caso de siringobulbia y consideraciones sobre tres casos operados del sistema nervioso ${ }^{9,23}$. De personalidad ciclotímica, la actividad de D. Ricardo se truncó prematuramente debido a su trágico fallecimiento.

El Dr. Arrazola comenzó a atender enfermos en la Clínica San Antonio y su amigo, el gran cirujano D. Manuel Cárdenas Rodríguez, lo introduce en la sociedad médica donostiarra presentándole como "el mejor neurocirujano del mundo", consiguiendo entrar en el Hospital de la Cruz Roja allá por 1953. En dicha Institución incorpora, no sin dificultades, las técnicas asépticas, antisépticas, neurorradiológicas y quirúrgicas aprendidas en Utrecht, entre ellas la cirugía de la hernia discal, hasta entonces desconocida en nuestro medio. En el $8^{\circ}$ Congreso Europeo de Neurocirugía que organizó el Dr. Fabián Isamat de la Riva en Barcelona (1987), tuve el honor de ayudar a mi jefe a recopilar casos y firmar junto a él una comunicación oral sobre su experiencia personal en 2550 pacientes intervenidos de hernia discal lumbar ${ }^{2}$. Trabajador infatigable, mi maestro compagina su inicial quehacer neuroquirúrgico entre San Sebastián y Bilbao, donde acude muchos viernes de cada mes durante dos años consecutivos. Operaba en el Hospital de Basurto (Bilbao), donde el Prof. Piniés le pide que se quede como Jefe de Servicio, pero declina la oferta, estableciéndose definitivamente en San Sebastián, donde forma parte del cuadro médico del Hospital Provincial y de la Residencia Sanitaria desde su inauguración.

Permítame el lector que introduzca un apartado que puede aclarar la evolución de la estructura hospitalaria en la ciudad de San Sebastián durante el siglo XX:

\section{Marco geográfico e histórico hospitalario de San Sebastián}

Aunque considero que la Historia la hacen las personas y no los Centros o las Instituciones, creo necesario hacer una pequeña crónica sobre la Medicina Hospitalaria
Donostiarra. Para ello resumo la documentación aportada fundamentalmente por los Dres. Munoa y Urkia ${ }^{23}$ : Puede decirse que en la ciudad de San Sebastián, desde principios del Siglo XX hasta la década de los sesenta, la asistencia sanitaria se caracterizó por el incremento y desarrollo de clínicas privadas que denotan el auge de la burguesía que puede permitirse el pago de estos centros. Tampoco se puede olvidar que la ciudad es centro de veraneo de gran renombre, lo que hace que las celebridades médicas vengan a la ciudad y atraigan una importante y a veces aristocrática clientela que mantiene estas clínicas. Entre ellas podemos destacar las construcciones de la clínica "villa San Ignacio" (inaugurada en 1906), clínica del Perpetuo Socorro (1908), clínica Nuestra Señora de las Mercedes (1913), clínica Nuestra Señora de Aránzazu (1921), clínica de San José (1922), clínica San Antonio (1928), Sanatorio Quirúrgico del Doctor Martín Santos (1936), clínica del Pilar (inaugurada el 14 de Abril de 1947) y clínica del Coro (fundada en 1947). Dentro de ellas ejercían diferentes especialistas, predominando internistas, cirujanos, traumatólogos, oculistas, ginecólogos, urólogos, otorrinolaringólogos... Otras Instituciones Sanitarias como el Hospital de la Cruz Roja fue inaugurado el 1 de Octubre de 1930 en recuerdo de la Reina María Cristina, que había fallecido meses antes y auténtica promotora de su construcción. Sita en la calle Matía y cerca del Palacio de Miramar del barrio del "Antiguo", la Familia Real en pleno acudió al acto: Alfonso XIII y Dña. Victoria Eugenia, los infantes, el Alcalde Juan José Prado y demás autoridades. El Instituto Radio-Quirúrgico de Guipúzcoa, inaugurado el 13 de Agosto de 1933, fue el segundo centro anticanceroso de España. Se ubica en los terrenos de Aldaconea, tiene un aspecto de casa vasca y sus promotores fueron los Dres. Luis Ayestaran y Benigno Oreja. Pertenece desde 1952 a la Caja de Ahorros de Guipúzcoa y es rebautizado en 1957 con el nombre de Instituto Oncológico, tal como hoy se le conoce. Su emplazamiento está a punto de desaparecer y trasladarse a un moderno edificio actualmente en construcción, en el alto de Zorroaga, adyacente al Hospital Donostia.

La actual ubicación del "Complejo Hospitalario" se decide en 1932. La Diputación y el Ayuntamiento de San Sebastián, que forman parte de la Junta de Beneficencia, acuerdan edificar un nuevo Hospital para la capital y su Provincia, ubicado en el alto de Zorroaga una colina frente al mar y de espaldas a la montaña desde la que se domina toda la ciudad. Las obras se iniciaron con un presupuesto de doce millones de pesetas, hasta que en 1936, la guerra Civil paralizó su construcción en su estructura de hormigón, viendo posteriormente construirse en esa misma zona el Sanatorio Psiquiátrico en 1944 y el Hospital de enfermedades del tórax en 1953, este último dependiente del Patronato Nacional de Lucha Antituberculosa se inaugura oficialmente el 12 de Agosto de 1953. 


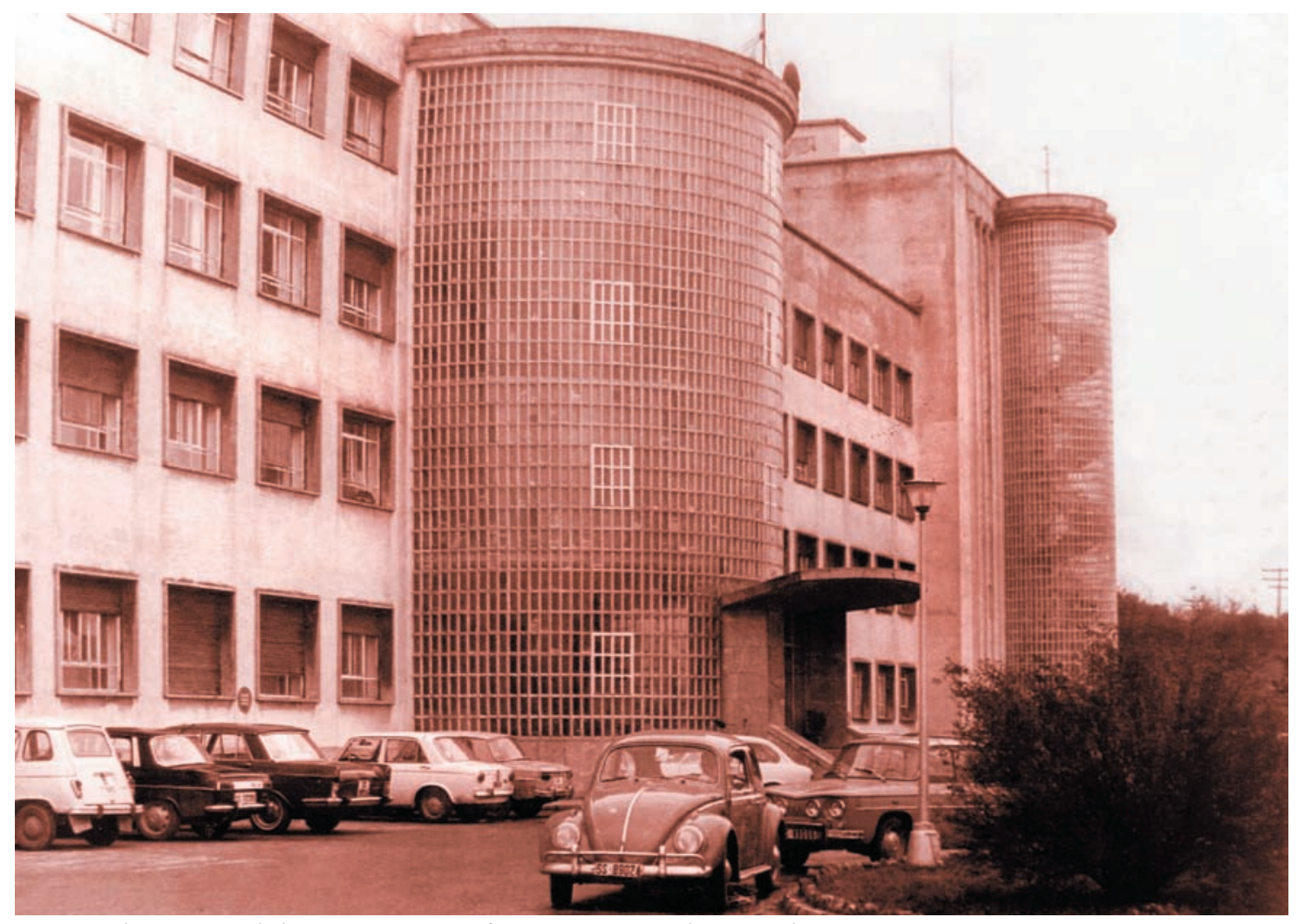

Figura 1. Hospital Provincial de Guipúzcoa en funcionamiento después de su inauguración en 1960.

El pabellón previsto para Escuela de Enfermeras se decide dedicarlo a Hospital Provincial, diseñado por el arquitecto Muñoz Baroja, con 156 camas, siendo inaugurado el 19 de Marzo de 1960 (Fig.1). La Dirección del Centro correspondía administrativamente al psiquiatra D. Luis Martín-Santos, director del sanatorio psiquiátrico, sin embargo, se optó por otro profesional, internista de gran prestigio y con capacidad de gestión: el Dr. D. Carlos Elósegui. Se respetaron las Jefaturas de Servicio provenientes del viejo Hospital de Manteo, como oftalmología (Dr. Azcoaga) y laboratorio (Dr. Irizar). El Sanatorio Psiquiátrico y este nuevo edificio de reciente construcción, dependientes ambos de la Diputación Foral, constituirán el Hospital Provincial de Guipúzcoa.

Por otra parte, el Instituto Nacional de Previsión se planteaba desde los años cincuenta construir un nuevo centro sanitario en San Sebastián. Para ello se puso en contacto con la Diputación, propietaria de los terrenos en los que, 20 años antes, se habían iniciado las obras de lo que iba a ser el Hospital de San Sebastián y que quedaron paralizadas en su chasis por la guerra civil: sobre la estructura central del proyectado Hospital Provincial, reducido al esqueleto de hormigón, se comenzó a construir la Residencia Sanitaria. Las obras progresaron hasta su construcción definitiva: la Residencia Sanitaria del Seguro Obligatorio de Enfermedad, bautizada con el nombre de Nuestra
Señora de Aránzazu e inaugurada el 15 de Agosto de 1960, 5 meses después del Hospital Provincial. Con una dotación inicial de 650 camas distribuidas en cuatro plantas y con un techo de pizarra que la caracteriza, siendo su primer Director el Dr. D. Alfonso Ugalde. Tiene acreditación docente desde 1975. En 1985 se construye por detrás de la Residencia Sanitaria el edificio Materno Infantil, dependiente del Insalud.

Así pues, desde el año 1960 existieron 3 centros hospitalarios públicos muy cercanos entre sí, geográficamente localizados en el alto de Zorroaga, atendiendo a la población desde organismos distintos; dos de ellos eran considerados como hospitales generales (Hospital Provincial y Residencia Sanitaria Nuestra Señora de Aránzazu) y el tercero, el Hospital del Tórax (antiguo antituberculoso) (Fig.2). El Hospital Provincial junto con el psiquiátrico dependiente de la Diputación Foral, la Residencia Sanitaria perteneciente al Insalud y el Hospital del tórax perteneciente al Patronato Nacional de la lucha antituberculosa ${ }^{6}$. Hasta entonces, la Sanidad pública en San Sebastián apenas contaba con el Hospital Civil de San Antonio Abad u Hospital de Manteo, inaugurado el 20 de Enero de 1888, festividad de San Sebastián, situado en el barrio de Gros, en la falda del monte Ulía y derribado tras la construcción del Hospital Provincial de Guipúzcoa. Con los distintos decretos de transferencias, los tres centros hospitalarios 


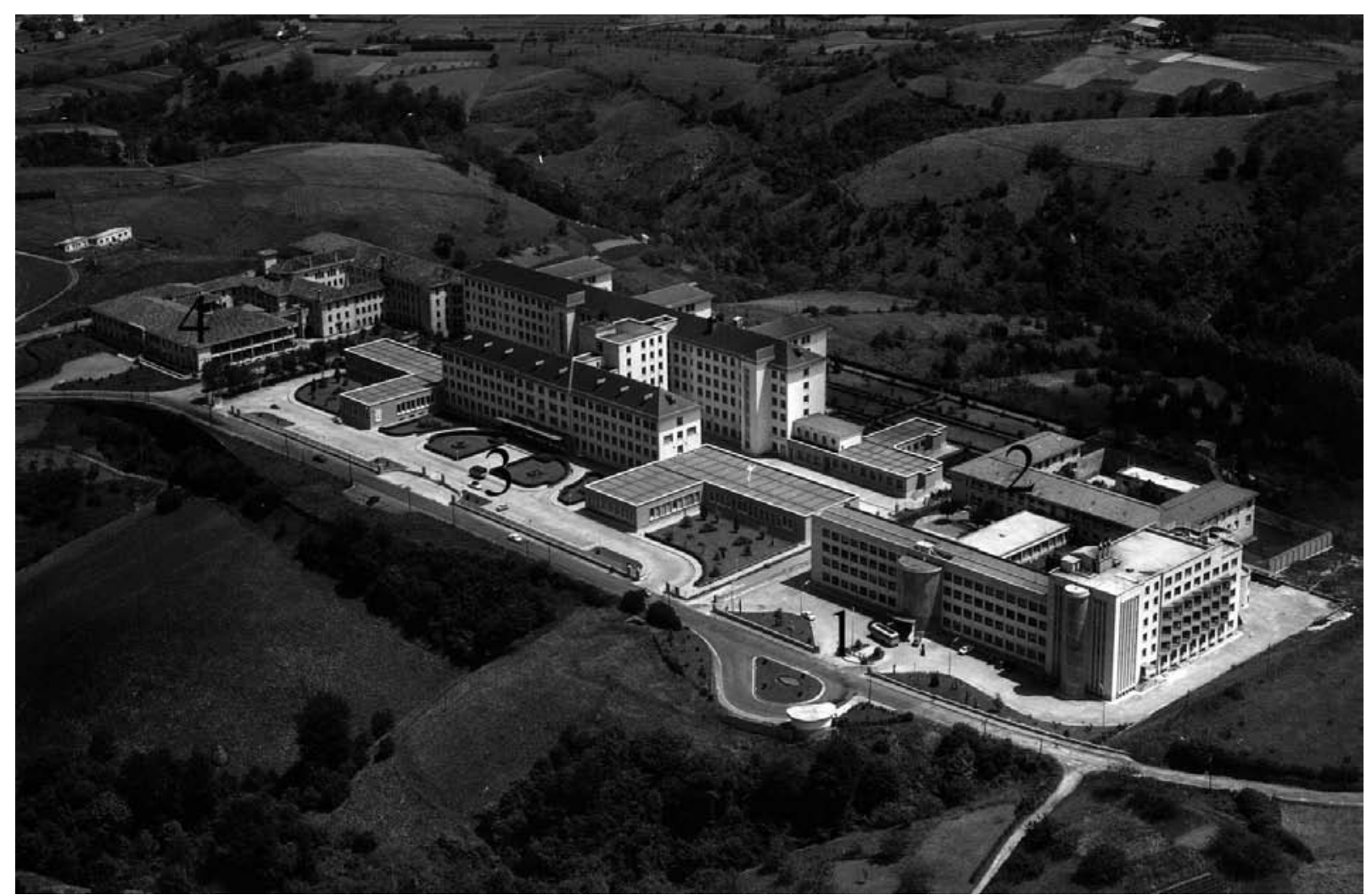

Figura 2. Hospitales del alto de Zorroaga en 1965. Leyenda: 1: Hospital Provincial. 2: Sanatorio Psiquiátrico. 3: Residencia Sanitaria. 4: Hospital del Tórax.

pasan a formar parte de la red Osakidetza/Servicio Vasco de Salud.

En 1981 el Hospital del Tórax se transfiere al Gobierno Vasco y en 1985 recibe el nombre de Hospital de Amara. Con los años, la disminución de tuberculosis hace que el Hospital dirija su atención al resto de las patologías respiratorias, quedando posteriormente el centro orientado a Hospital de media y larga estancia. El Hospital Provincial pasa a integrarse en 1985 a la red hospitalaria Osakidetza como centro de agudos, atendiendo, con el nombre de Hospital de Guipúzcoa a una comarca sanitaria formada por 15 municipios y una población de 160.000 habitantes. La Residencia Sanitaria Nuestra Señora de Aránzazu, centro hospitalario de carácter terciario, se integra en la red sanitaria vasca con el decreto de transferencias de 1987, recibiendo el nombre de Hospital Aránzazu. Esta nueva situación de los Hospitales del alto de Zorroaga transferidos a Osakidetza/Servicio Vasco de Salud, abren un nuevo periodo de integración de los diferentes Servicios Clínicos, que en general se han conocido con el nombre de Plan Zorroaga ${ }^{6}$. En 1985 se construye un nuevo edificio Materno-Infantil, con el trasvase físico de los Servicios de Pediatría, Ginecología y Obstetricia tanto de la Residencia Sanitaria como del Hospital de Guipúzcoa.

La proximidad física, la titularidad única, las sinergias de la gestión sanitaria moderna y una voluntad política en este sentido, hacen que en Diciembre de 1995 salga a la luz un borrador con el título "plan estratégico de los Hospitales Públicos de Donostia y estructura organizativa del Complejo Hospitalario Donostia", diseñando un modelo abierto de estructura organizativa, que no era otra cosa que tratar de evitar duplicidades y abaratar costos, es decir: integración en busca de una mejora en la calidad asistencial. Aunque físicamente tan cercanos que se dan la mano, no hay que olvidar que los 3 hospitales públicos están clásicamente separados por culturas paralelas, se buscó un consenso que beneficiara a las partes implicadas: profesionales y Organización Sanitaria en conjunto. El 22 de Abril de 1996 se inauguran unos puentes de conexión entre los Hospitales de Guipúzcoa, Amara y Aránzazu, pivotando sobre este último el eje central: la primera de las actuaciones que hacen visible la unión. Con la idea de "combinar eficazmente las ventajas organizativas de lo grande con las ventajas que provienen de la gestión de lo pequeño", se crea el 22 de Mayo de 1997 el "Complejo Hospitalario Donostia", estableciéndose una figura administrativa y de gestión nueva, inexistente hasta entonces, como es la de Gerente del Complejo, recayendo el nombramiento en D. José Antonio Aguirre Elustondo. Son épocas de cambios y el primero de ellos correspondió a un Servicio Central 


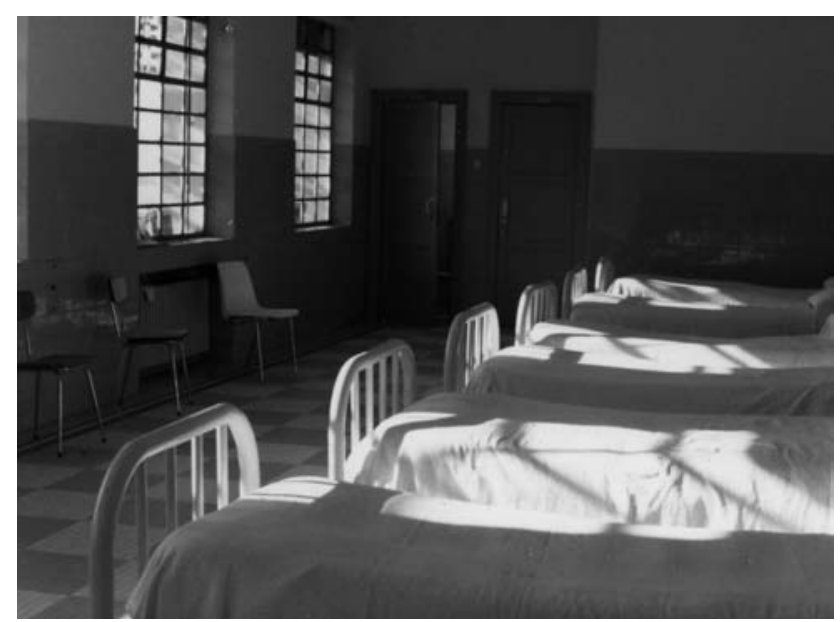

Figura 3. Sala de ingresos del Sanatorio Psiquiátrico.

como fue el de Laboratorio, inaugurándose oficialmente con el nombre de "Laboratorio Unificado de Donostia" el 25 de Mayo de 1998. Estos cambios afectarán también a los Servicios Clínicos como los de anatomía patológica, cirugía vascular, radiología, neurocirugía... y en Mayo del 2000 se inicia una nueva andadura neuroquirúrgica tras la fusión de los dos Servicios.

Por acuerdo del Consejo de Administración del Ente Público Osakidetza/Servicio Vasco de Salud, se crea el 14 de Febrero de 2001 el Hospital Donostia, suprimiendo las organizaciones de Servicios Sanitarios integrados en el anterior "Complejo Hospitalario Donostia", nombrándose para esta nueva etapa al Dr. Juan José Múgica Gerente del Hospital Donostia (último Gerente del Hospital Provincial de Guipúzcoa). Esta evolución de las estructuras sanitarias públicas donostiarras ha ido modelando también a los diferentes Servicios Clínicos, no menos al que nos atañe como es el de Neurocirugía en el Hospital Provincial, al cual dividimos de forma arbitraria en dos etapas:

\section{La neurocirugía en el Hospital Provincial: primera etapa (1960-1980)}

El Hospital Provincial se inaugura oficialmente el 19 de Marzo de 1960. En su estructura arquitectónica destacan en la fachada principal dos grandes cristaleras en forma de semicírculo, similar a la del Hospital de Cruces de Bilbao (Fig.1). Es un edificio moderno para su época y bien dotado, tiene cuatro plantas y habitaciones de no más de cuatro camas en cada una, desapareciendo las antiguas salas de ingreso con camas en hileras, como las que aun mantenía el Psiquiátrico (Fig.3). Además cuenta con habitaciones para médicos internos, escuela de enfermería y Comunidad para religiosas que cuidan enfermos, organizan los quirófanos y supervisan las plantas de ingreso hospitalario. Su primer Director (1960-1968) fue D. Carlos Elósegui, un prestigioso

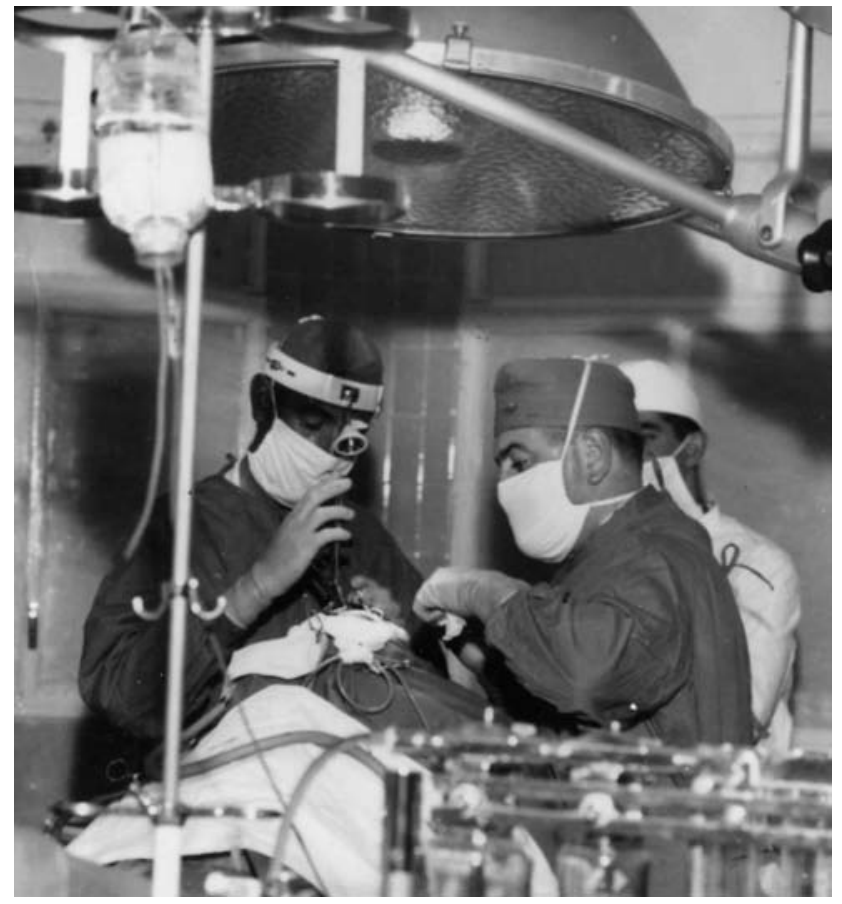

Figura 4. Los Dres. Arrazola y Díaz Aramendi operando en el recien estrenado quirófano del Hospital Provincial. 1960. (Fotografia cedida por el Dr. Díaz Aramendi).

internista que provenía, como otros destacados especialistas (Dres. Ansa, Azcoaga, Olarreaga...), del viejo Hospital de Manteo. Reputados cirujanos como Carlos Sanz, Mario Senra, Jose Maria Odriozola o Manuel Cárdenas Rodríguez completan el cuadro médico inicial.

El Dr. D. Mariano Arrázola Silio, ya por aquel entonces bien conocido y asentado en la ciudad como experto neurocirujano, tras demostrar sus dotes clínicas y quirúrgicas con excelentes resultados, fundamentalmente en el Hospital de la Cruz Roja, comienza a ver enfermos y operar en el Hospital Provincial, inaugurando sus quirófanos (Fig. 4). La primera intervención quirúrgica se trató de una leucotomía, tuvo como ayudante al Dr. Antonio Díaz Aramendi, de anestesista al Dr. Eguiguren y como practicantes a Jesús Elorza y Juanita Tellería (Fig. 5). No es de extrañar que fuera un caso de psicocirugía la primera intervención neuroquirúrgica que realizara mi maestro en el Hospital Provincial: las relaciones entre la Psiquiatría, Neuropsiquiatría y Neurocirugía eran estrechas como lo eran sus cabezas visibles: Luis Martín-Santos (1924-1964) era el Director del Hospital Psiquiátrico de San Sebastián y Jefe de los Servicios Psiquiátricos de la Diputación de Guipúzcoa desde que ganó las oposiciones en 1951 con 26 años de edad y el joven Javier Urcola Echeverría, recién incorporado como becario interno por oposición en 1961 en el Servicio de Neurología y Psiquiatría del Hospital Provincial, con quienes mantenía una estrecha relación 


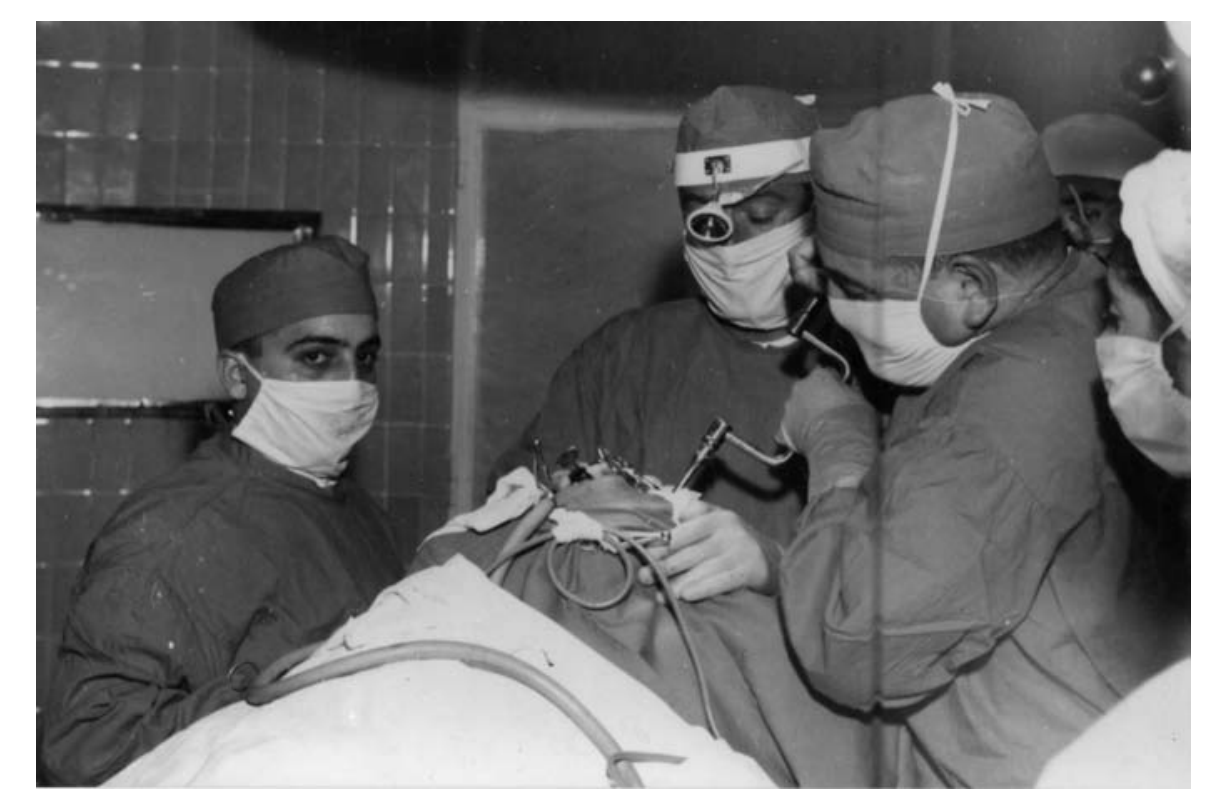

Figura 5. El Dr. Arrazola (con fotóforo) y Diaz Aramendi (con el trépano de mano) realizando la primera intervención neuroquirúrgica del Hospital Provincial de Guipúzcoa, como fue una leucotomía. 1960. (Fotografia cedida por el Dr. Díaz Aramendi). profesional y personal. Siguiendo al neurólogo Javier Olascoaga, puede decirse que entre los tres crearon la primera Unidad de Neurociencias del País Vasco.

Esta primera etapa es una época difícil de inicio y expansión de la neurocirugía, para lo cual mi maestro estaba bien preparado después de su formación en Holanda. Se configuran y organizan los equipos. No existían Scanner, microscopio quirúrgico ni aspiradores ultrasónicos, la coagulación era monopolar ("Bovie"), el diagnóstico se basaba mucho en la clínica y menos en la imagen radiológica, que por cierto realizaba el neurocirujano con mucha imaginación, audacia y sin ayuda, apoyándose en la mielografía, arteriografía, ecografía cerebral, ventriculografía, neumoencefalografía... (Fig.6). No hay que olvidar que durante los sesenta se seguía utilizando el trépano de mano y la sierra de Gigli para las craneotomías, la iluminación durante la cirugía se basaba en las lámparas colgadas del techo y del fotóforo (que no era otra cosa que una pesada luz frontal con una bombilla eléctrica que se recalentaba, recordando a la luz que usaban los mineros), las suturas eran de hilo de seda que estaba enrollado en un carrete que se reesterilizaba y que la instrumentista cortaba a la misma longitud y enhebraba con una agujas de diferentes tamaños, la misión del primer ayudante era la de hacer un buen campo con las espátulas que sujetaba con una mano, con la otra echaba gotas de suero con una pera y con un pie pisaba el pedal de la coagulación. Los

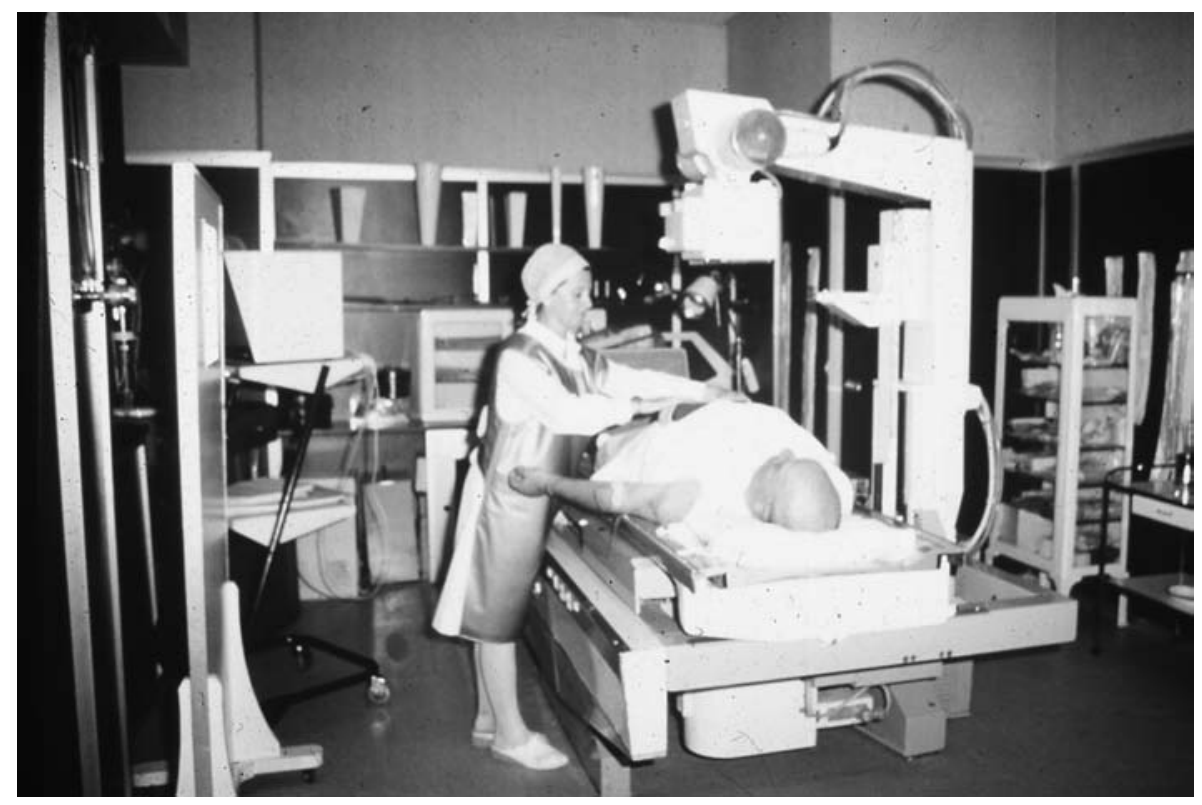

Figura 6. Sala de radiología del Hospital Provincial con mesa basculante. 


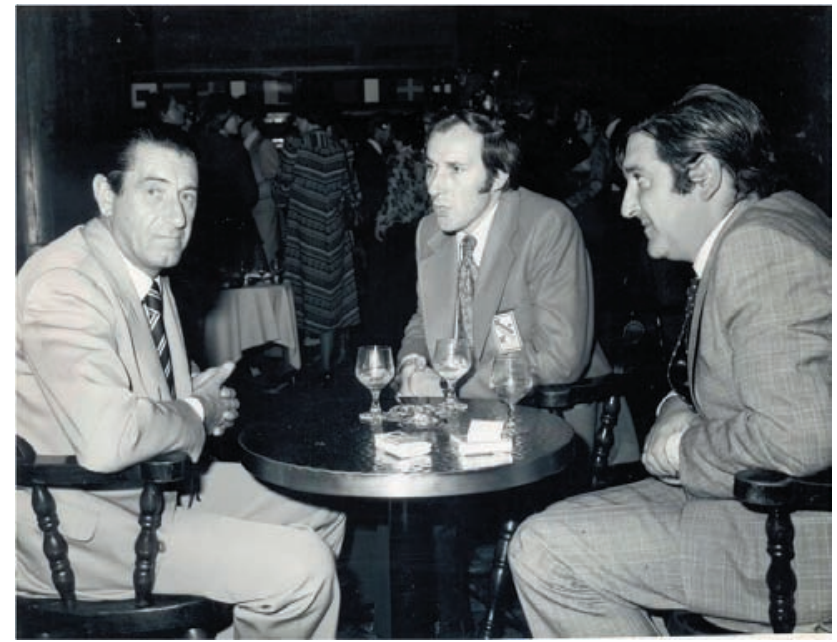

Figura 7. El Dr. Arrazola (izquierda) sentado junto a los Dres. Javier Urcola (derecha) y Moseley (en el centro). Londres 1977.

esfuerzos del anestesista no conseguían a veces frenar el edema cerebral que hacia ascender como un globo al cerebro a través de los bordes de la duramadre abierta, convirtiendo la cirugía en un drama y la craneotomía en una craniectomía descompresiva. No existía formación MIR y para obtener el título de especialista bastaba con apuntarse en el colegio de Médicos y dejar correr unos años. En 1960 y organizada por el Dr. Albert, la Sociedad Luso-Española de Neurocirugía celebró su reunión en Sevilla, entrando a formar parte como Socios los Dres. Bravo Zabalgoitia, Ley Palomeque, Martínez Pastor, Revuelta Gutiérrez y Sanjuan Benito: apelados de forma cariñosa "los jóvenes coroneles" por D. Máximo Poza y Poza ${ }^{17}$.

El Hospital Provincial se nutría fundamentalmente de pacientes provenientes de la Beneficencia y de Seguros particulares como Diputación, Lagun Aro y otras patronales de trabajo. Algunas empresas potentes guipuzcoanas tenían un concierto económico con el Hospital para atender a sus empleados, disponiendo para dichos pacientes una planta de ingresos llamada de "privados". Realmente no existía en la Neurocirugía del Hospital durante esta época, una estructura de Servicio como tal con formación de Residentes, Sesiones Clínicas, asignación de camas o quirófanos etc. Sino que era la figura de mi maestro, una neurocirugía muy personal, que acudía cuando se le requería (siendo lo que actualmente conocemos como neurocirujano consultor), con diferentes ayudantes que provenían fundamentalmente de los médicos Internos del Hospital Provincial, entre ellos figuraron progresivamente Eugenio Lasheras, Juan Erasun, Arturo Sánchez Camino y José María Zapirain. O, como durante los primeros años, con Antonio Díaz Aramendi, quien le ayudaba asimismo en otros centros como en el Hospital de la Cruz Roja. En aquella época, la figura de "ayudante" era un título de gran mérito y aun mas serlo del Dr. Arrazola, quien tenía también otro equipo de ayudantes en la "casa grande" (Residencia Sanitaria) como Gómez Taborga y Angel Ramos (quienes posteriormente se trasladan a Burgos).

Aunque ya ejercía las funciones de Jefe tanto en la Residencia como en el Hospital Provincial, Don Mariano ganó las oposiciones de la Jefatura de Servicio en el Seguro Obligatorio de Enfermedad con el numero uno y firmó el 1 de Mayo de 1966 su primer contrato como Jefe del Servicio de Neurocirugía en la Residencia Sanitaria Nuestra Señora de Aránzazu. Hace años me contaba el Dr. Izquierdo una anécdota que puede aclarar la situación incipiente de la Neurocirugía Hospitalaria: en el verano de 1968 vino José $M^{\text {a }}$ Izquierdo Rojo a San Sebastián destinado para hacer las prácticas de su Servicio Militar como Alférez de Complemento al cuartel de Loyola ${ }^{11}$ y quiso presentarse a Don Mariano, que fue amigo de su padre (el neurocirujano asturiano D. José Ma Izquierdo Rubín), para asistir a las actividades del Servicio de Neurocirugía en sus ratos libres. Al preguntar por Don Mariano, tanto en la Residencia Sanitaria como en el Hospital Provincial no sabían decirle en realidad donde estaba: de un Hospital le mandaban al otro, haciendo la misión imposible y desistiendo de encontrarle, finalizó sus prácticas Universitarias Militares sin conocer ninguna sesión neuroquirúrgica donostiarra: probablemente coincidiría con alguna expedición montañera de mi maestro. Dentro de las intervenciones mas arriesgadas y no tan infrecuentes que realizaba el jefe durante los años setenta en el Hospital Provincial, eran los aneurismas cerebrales, recordando nuestro antiguo y querido anestesista Antonio Palacios Rubio, los madrugones que se pegaba para llegar a las seis de la mañana y conseguir con antelación suficiente a la cirugía una hipotermia moderada de $31^{\circ}$ $\mathrm{C}$ a base de cubitos de hielo que traía el practicante desde la cocina y aplicaban al paciente en axilas, ingles... junto con una manta de agua fría. En 1970 organizó con gran éxito, el Congreso de la Sociedad Luso-Española de Neurocirugía junto con la Sociedad Francesa de Neurocirugía en San Sebastián. En este periodo un grupo de médicos, entre ellos mi jefe, inician contactos y reuniones con empresarios para la creación de un nueva clínica privada que llamarán Policlínica Guipuzcoa, inaugurándose en 1975. Fue tras la Clínica Ruber de Madrid, la primera en incorporar la nueva y revolucionaria técnica diagnóstica de la tomografía axial computarizada con la adquisición del Emi-Scanner. Para ello, Don Mariano, junto con los Dres. Munoa, Gabilondo y Urcola entre otros pioneros, deciden adquirir el nuevo Scanner de la Compañía EMI y establecen contacto con Moseley en Londres (Fig.7), incorporando a la recién creada Policlínica Guipúzcoa la nueva Unidad de Diagnóstico en 1976. Ya para entonces, el grupo de D. Sixto Obrador publica la primera serie de cien enfermos neuroquirúrgicos 


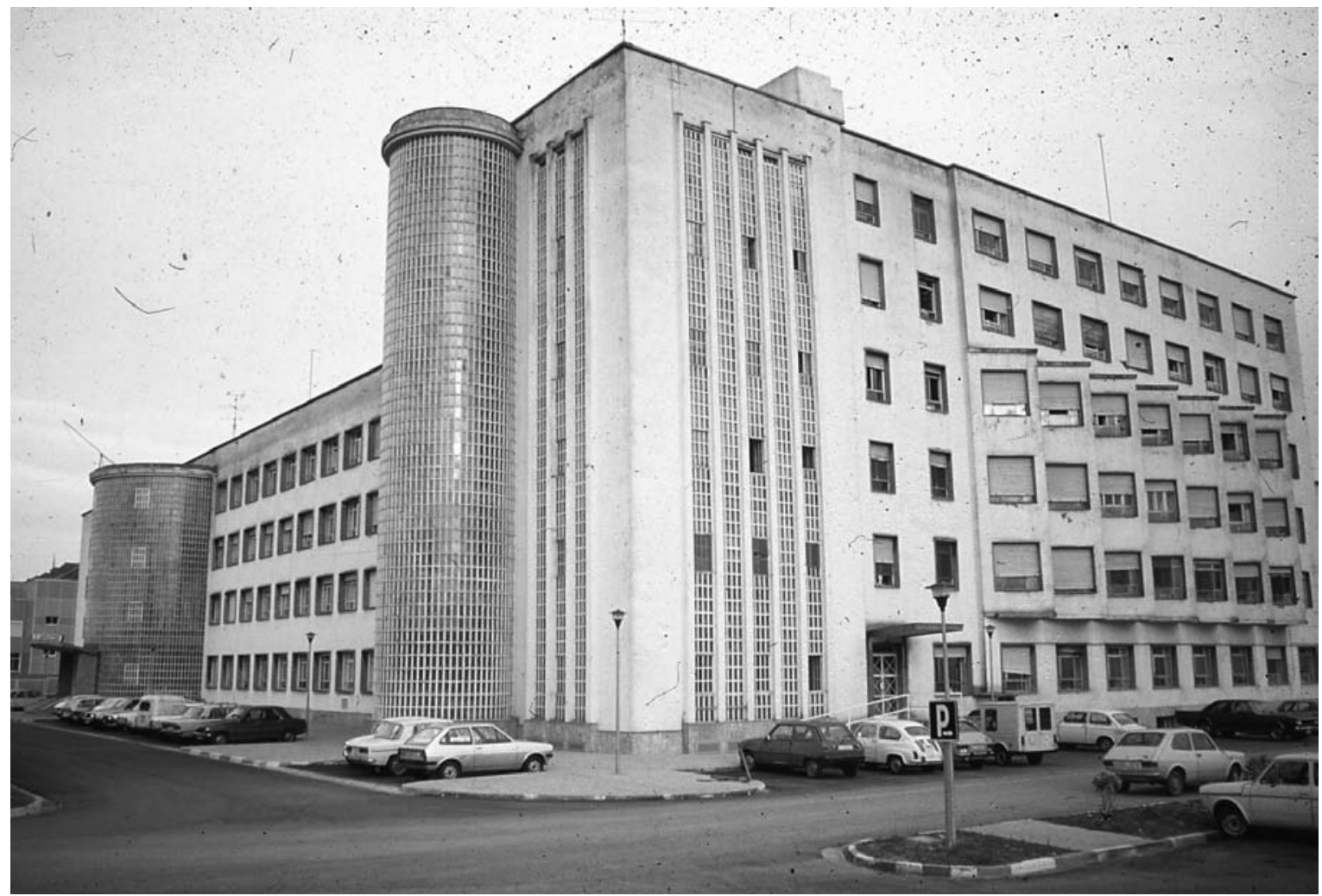

Figura 8. Fachada principal del Hospital Provincial en 1982.

estudiados con tomografía axial computarizada ${ }^{16}$.

Es a finales de esta época cuando el que suscribe entra a formar parte del Servicio de Neurocirugía de Arrazola después de conseguir plaza en la Residencia Sanitaria Nuestra Señora de Aránzazu como Residente de Neurocirugía en la convocatoria MIR del año 1977. Venía de Madrid después de estar durante los últimos tres años de mis estudios de Medicina como alumno interno en el Servicio de Neurocirugía de D. Pablo Peraita Peraita. Tomé posesión de la plaza como "Residente 1" el 13 de abril de 1978. Curiosamente dos semanas después de tomar posesión de la plaza, fallecía en Madrid un gran amigo del jefe como era D. Sixto Obrador Alcalde (1883-1978), ahora puedo comprender el estado de ánimo de mi maestro cuando me presenté a él en su despacho como nuevo residente y la reacción de sorpresa que le causé, ya que el no había solicitado ningún residente. Pero no debí de caerle tan mal como yo pensaba, pues de forma prácticamente inmediata tuve la suerte de comenzar a ayudarle en todas sus actividades fuera de lo que se suponía mi estricta formación como residente dentro de la Residencia Sanitaria Nuestra Señora de Aránzazu, incluyendo por supuesto el Hospital Provincial. Colaborábamos con el jefe en la atención del paciente neuroquirúrgico del Hospital parte del equipo de la Residencia: Arturo Sánchez Camino, José María Zapirain, Benito Navajas, Guillermo Rejas y yo mismo, donde a veces el jefe nos pagaba de su bolsillo un sobresueldo que nos venía muy bien a todos. Tuve la fortuna de ser su ayudante, junto con Arturo Sánchez Camino, en muchas cirugías discales, craneales y de nervios periféricos realizadas a las tardes (o algún sábado a la mañana) en el Hospital Provincial. Recuerdo sobre todo los últimos casos de leucotomías en enfermos mentales y el pase de visita durante los siguientes días en el psiquiátrico para valorar el postoperatorio, con un celador que nos guiaba ahuyentando a quienes osaran acercarse a nosotros por los pasillos, llevando un gran manojo de llaves con las que abría y cerraba unas grandes puertas que crujían a nuestro paso.

\section{La neurocirugía en el Hospital Provincial: Segunda etapa (1980-2000)}

Epoca de consolidación y auge tecnológico de la neurocirugía, época de cambios drásticos en la Gestión Hospitalaria, con la aparición en 1980 de una nueva figura como fue la de Director Gerente del Hospital Provincial en la persona de D. Baltasar Marín. Tradicionalmente hasta entonces habían existido en el Hospital Provincial Directores Técnicos, siempre Médicos de gran categoría profesional como Carlos Elósegui, Jesús Olarreaga y Angel Olaso. El desarrollo de la jerarquización impulsada por el nuevo Gerente introdujo nuevos cambios en la estructura hospita- 


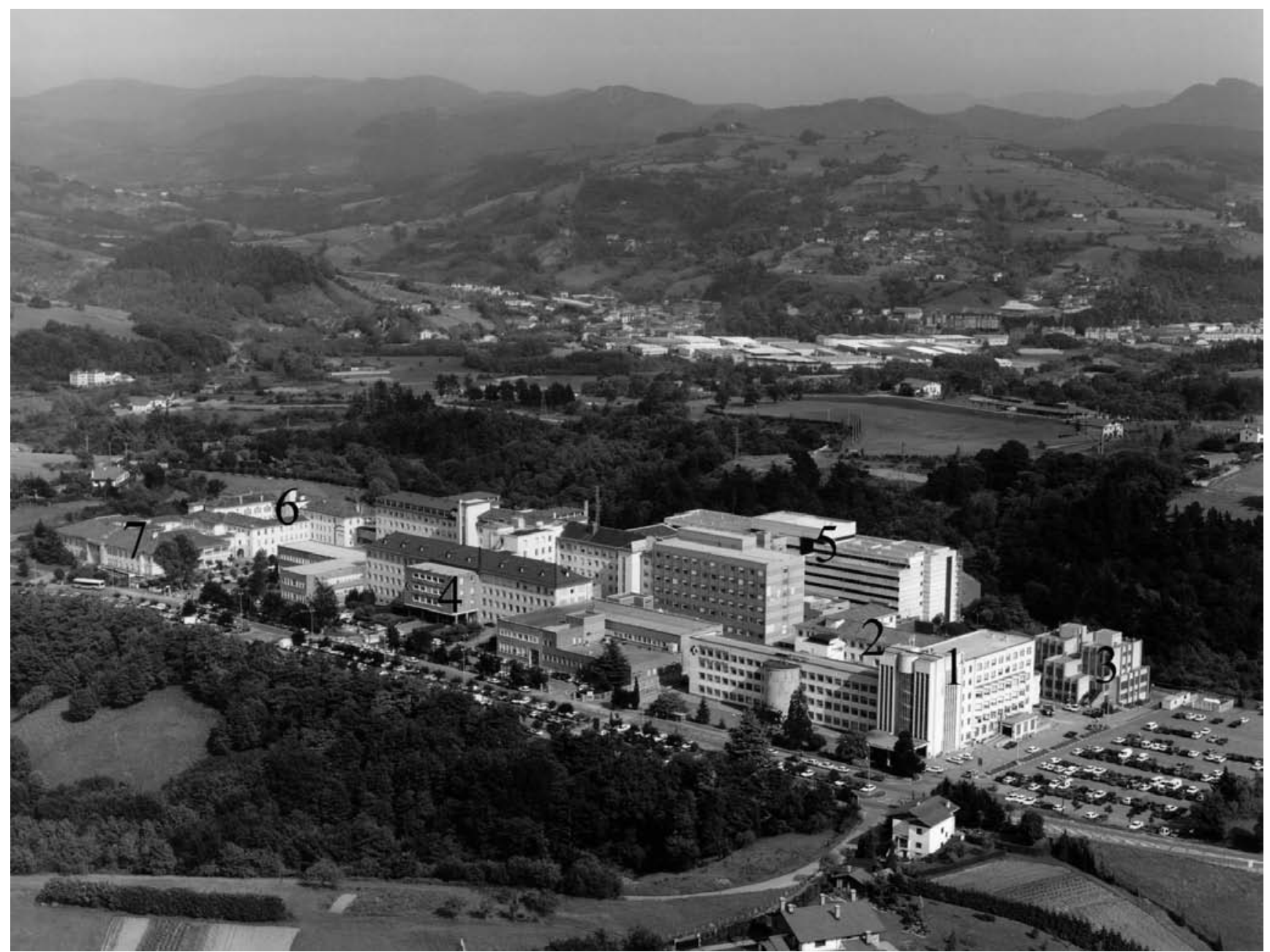

Figura 9. Vista general de los Hospitales en el alto de Zorroaga en 1998. Leyenda: 1: Hospital de Guipúzcoa. 2: Psiquiátrico. 3: Villa Marín. 4: Hospital Aranzazu. 5: Hospital Materno-Infantil. 6: Hospital del Tórax. 7: Escuela Universitaria de Enfermería y Unidad Docente de Medicina.

laria, los grandes Servicios como Medicina Interna, Cirugía General, Laboratorio, Pediatría, Radiología, Oftalmología... contaban con Jefes de Servicio que habían obtenido la plaza por concurso-oposición o por contratación como Antonio Palacios, jefe de anestesiología y reanimación. Las especialidades médicas y quirúrgicas como neurología, endocrinología, neumología, otorrino, cirugía plástica, urología, neurocirugía... tenían categoría de Secciones dentro de grandes Servicios como eran Medicina Interna y Cirugía General con Gabriel Zubillaga y José Ramón Ribera como Jefes de Servicio respectivamente. Desapareció progresivamente la práctica privada dentro del Hospital o el pago por acto médico, se consolidaron Secciones y Servicios Clínicos, se fomentaron Sesiones Clínicas y se controló el horario a todo el personal mediante fichaje con una tarjeta de identificación personal que de forma obligada debía llevarse en un lugar bien visible de la bata médica, cuestión que particularmente a mi no me preocupó, ya que lo había conocido en algunos hospitales americanos y no me importaba fichar. En boca del Gerente D. Baltasar Marín, estos drásticos cambios en la forma de gestionar el Hospital, no bien entendidos por todos, trataron de buscar unas señas de identidad que nos identificaran y diferenciaran de nuestro gran competidor como era la Residencia Sanitaria, entre ellos, la adquisición de un moderno angiógrafo digital para el Servicio de Radiología que lideraba José Antonio Recondo, ofreciendo un nuevo dimensionamiento e imagen de estructura hospitalaria (Fig.8). La construcción de un nuevo edificio junto al Hospital (que llamábamos y seguimos llamando "Villa Marín"), fue inaugurado en 1983 y supuso la culminación de esta renovación hospitalaria: allí fueron a parar los despachos de Gerencia, Dirección y Administración, junto con salas de reuniones, biblioteca, salón de actos, comedor de médicos, residencia de internos y comunidad de religiosas (Fig.9). Dicho edificio actualmente está siendo remodelado para convertirlo en centro de 


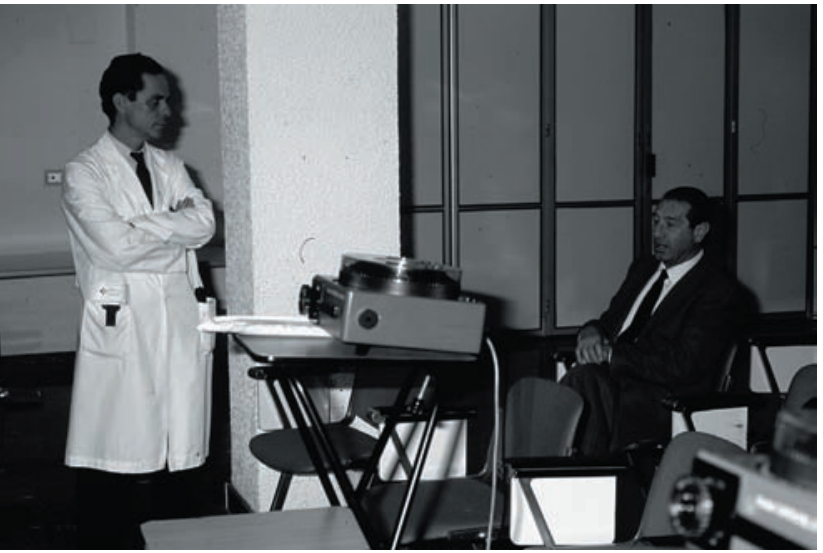

Figura 10. El Dr. Arrázola, sentado en primera fila, durante la sesión interhospitalaria "tratamiento de la neuralgia del trigémino". Salón de actos del Hospital de Guipúzcoa. 20.12.1993.

Docencia e Investigación del Hospital Donostia.

En 1981 ya había acabado los cuatro años de Residencia y con mi recién estrenado Título de Especialista en Neurocirugía seguía ayudando al jefe en el Hospital Provincial, donde tanto a Guillermo Rejas como a mí periódicamente nos hacían contratos como neurocirujanos de guardia (por supuesto localizada) o de sustitución durante las vacaciones del jefe y de Arturo Sánchez Camino (únicos miembros en plantilla de la Sección de Neurocirugía), hasta que en Julio de 1983 la incompatibilidad con el puesto de trabajo en la Residencia Sanitaria Nuestra Señora de Aránzazu, hizo que tanto el jefe como Arturo Sánchez Camino optaran por quedarse exclusivamente en "la casa grande" y me quedara yo solo como neurocirujano en el Hospital Provincial de Guipúzcoa con contratos renovables de Médico Adjunto Interino. Esta difícil y solitaria situación personal y profesional pude enjuagarla por el gran ambiente médico y excelentes relaciones que manteníamos los profesionales del Hospital, además seguía en relación con el Servicio de Neurocirugía de la Residencia donde había aprendido mis primeros pasos neuroquirúrgicos $\mathrm{y}$, por supuesto, atendiendo y ayudando a mi jefe en su consulta privada, quien siempre me animaba a resistir, aprendiendo a superar las dificultades: "usted aguante" me decía (nunca podré agradecerle suficientemente sus sabios consejos). Afortunadamente esta situación laboral inestable no se prolongó por mucho tiempo y en Septiembre de 1984 gané por Concurso Oposición, en la que también se presentó mi amigo Jesús Gómez Montoya, la plaza de Médico Adjunto de Neurocirugía del Hospital Provincial de Guipúzcoa. En este periodo, la sectorización hospitalaria de la población Guipuzcoana hacía que nuestro Hospital tuviera una mayor área de influencia, siendo Hospital de referencia sobre unos 160.000 habitantes, lo que hacía soportar a las recién remo- deladas secciones y servicios clínicos una importante carga asistencial que asumíamos sin escatimar esfuerzos.

A pesar de mi soledad nunca me sentí solo, en diferentes épocas y situaciones estuve ayudado por grandes amigos neurocirujanos sin los cuales no hubiera podido resistir y a quienes tengo que agradecer su empuje y entusiasmo como Ignacio Onzain Behobide, Mariano Arrazola Schlamilch, Luis Martínez Soto, Fernando Ugarriza Echebarrieta, Rafael Ramírez Penso y Roger Alfaro Baca. Asimismo, nuestra estrecha colaboración que llegó a ser de amistad personal, con neurólogos como Javier Urcola, Luis Guereca, Javier Olascoaga, Vicente Olasagasti, Ramón Zabalza o psiquiatras como Vicente Urcola, Ana Díaz Yarza, Crispín Batiz, Hilario Urbieta e Ignacio Querejeta, entre otros, hizo posible ir consolidando progresivamente la neurocirugía en el Hospital: aumentando el número de ingresos, intervenciones quirúrgicas, consultas, etc, mejorando anualmente nuestros índices de calidad en la actividad asistencial. Tanto fue así que comenzamos a tener asignadas 7 camas de ingreso en la planta de cirugía, llegando a realizar más de 150 intervenciones quirúrgicas anuales. Las inversiones eran cortas, pero suficientes para poder realizar cualquier tipo de intervención quirúrgica: recuerdo lo que nos costó convencer a la administración de la compra del primer fotóforo de luz fría o el primer microscopio "contraves" con posibilidad de documentación intraoperatoria (filmación y diapositivas), tratando de imitar, a finales de los ochenta, las recomendaciones que nuestro José Gerardo Martín Rodríguez describía a la perfección en el libro "Microneurosurgery" de R.W. Rand" ${ }^{14}$. Este último modelo de microscopio lo introdujimos gracias a la colaboración de mi amigo Julio Igartua, Jefe de ORL, con quien compartía mesa, mantel, quirófano $\mathrm{y}$, a veces, intervenciones quirúrgicas de la base craneal. Organizábamos charlas a los médicos internos y residentes, sesiones clínicas y reuniones -a veces interhospitalarias- como del tratamiento de los neurinomas acústicos, traumatismos craneoencefálicos, hemorragias subaracnoideas, neuralgia de trigémino... (Fig.10). El jefe ocasionalmente venía al Hospital para echarnos una mano, preparar alguna comunicación a congresos ${ }^{3}$, colaborar en algún caso, o bien organizar juntos algún acontecimiento, como la "XLI reunión de la sociedad luso-española de neurocirugía" a lo largo del año 1989. En 1994 recibimos, tanto el responsable de la Sección de urología, Iñaki Hernaez, como yo, el nombramiento de Jefe de Sección, lo que supuso un importante reconocimiento de nuestra actividad y un aldabonazo para ambas secciones. Dos años después, Mariano Arrazola Schlamilch conseguía por concurso oposición de la OPE (oferta pública de empleo), la plaza de médico adjunto de neurocirugía, asentando su posición y haciendo juntos un auténtico "tándem" con la ayuda de nuestras Pilar Viguera y María Jesús Díaz-Güemes, instrumentista y auxiliar res- 


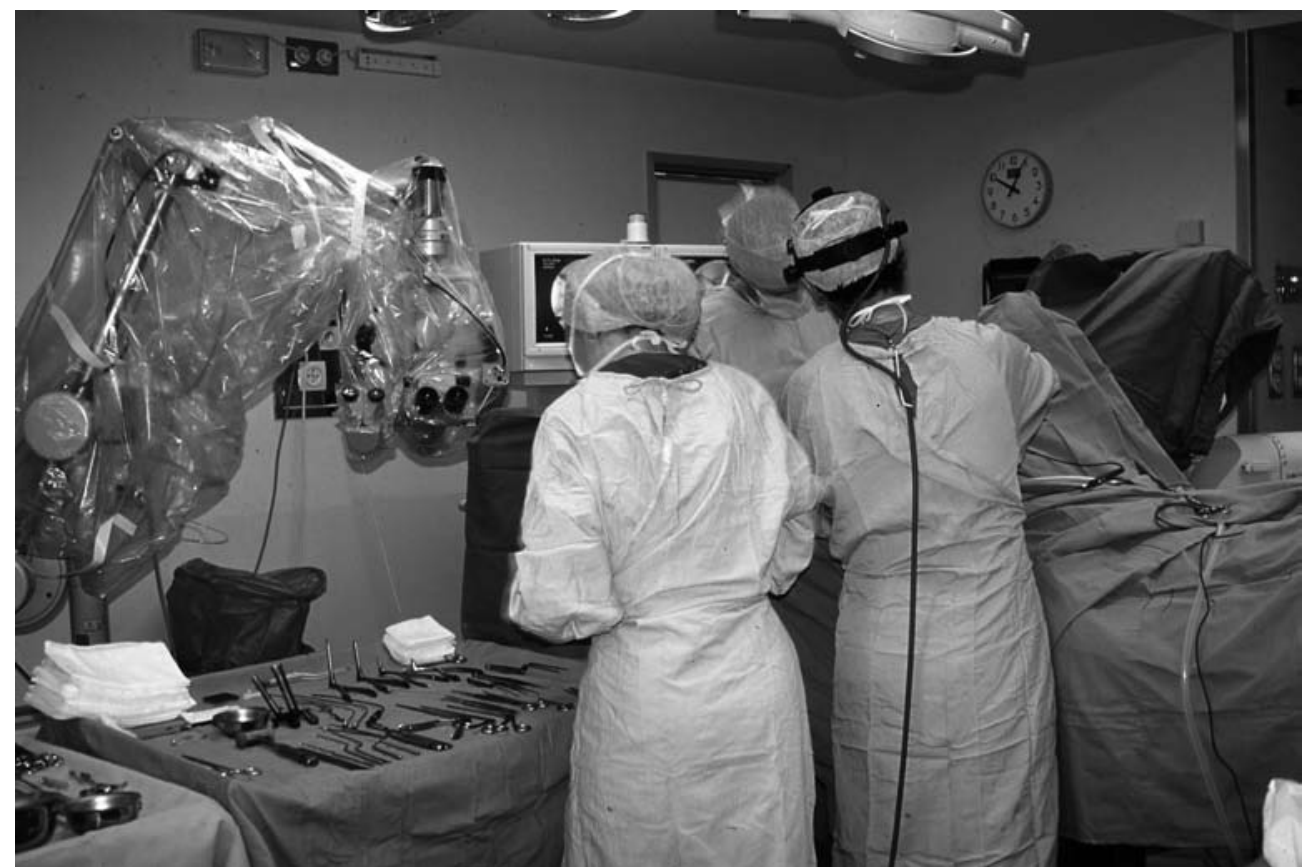

Figura 11. Quirófano de Neurocirugía del Hospital de Guipúzcoa en 1996: en frente el Dr. Arrazola Schlamilch y de espaldas nuestra instrumentista Pilar Viguera, junto a quien suscribe operando un adenoma de hipófisis por vía transesfenoidal.

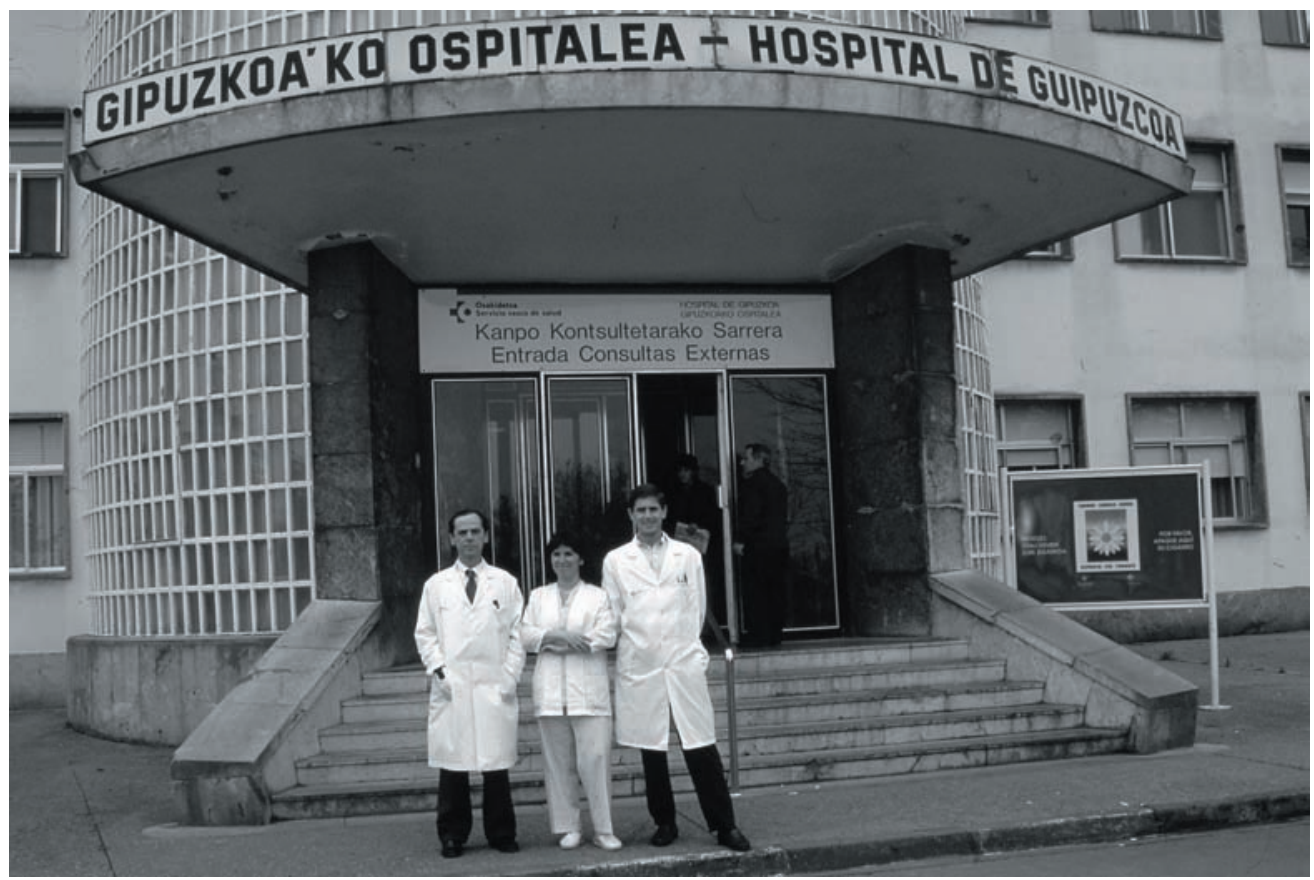

Figura 12. Entrada en consultas externas del Hospital de Guipúzcoa. En el centro: nuestra Auxiliar Maria Jesús DíazGüemes escoltada por Mariano Arrazola Schlamilch y yo mismo. Marzo 1997.

pectivamente (Figs. 11 y 12 ).

Era un pequeño Hospital con vida, agradable de vivirlo, nos conocíamos todos, no había horario, las consultas eran verbales, nos ayudábamos unos a otros, aprendimos en fin, sin saberlo, a trabajar en equipo. Aunque no es oro todo lo que reluce: hubo también sus dificultades, tensiones e incluso enfrentamientos, llegamos a superar situaciones difíciles, a veces comprometidas, que el paso de los años ha ido difuminando. Las relaciones con el equipo de neurología eran excelentes: no podré olvidar las charlas intermi- 


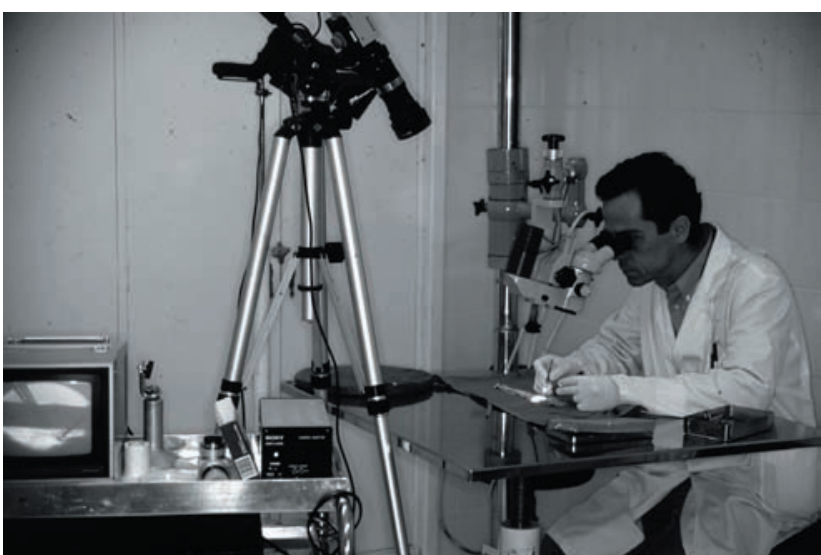

Figura 13. El autor en la mesa de microcirugía del laboratorio experimental (Hospital Provincial 1992).

nables con mi amigo Javier Urcola, Jefe de Neurología, a lo largo de los pasillos del Hospital, donde comentábamos casos, discutíamos indicaciones y resolvíamos problemas a veces complicados. Incluso nos daba tiempo para hacer trabajos y publicarlos conjuntamente de forma interdisciplinaria $^{18,22}$. El Servicio de Anestesia y Reanimación, liderado por Antonio Palacios, Begoña Basterrechea y posteriormente por Antonio Berbiela, colaboraba con nosotros facilitándonos la cirugía con la introducción de nuevas técnicas anestésicas en intervenciones microquirúrgicas raquimedulares o de base de cráneo, como la nucleotomía percutánea, microdiscectomía, descompresión neurovascular del V par o la microcompresión percutánea del ganglio de Gasser en el tratamiento de pacientes con neuralgia de trigémino. Esta última realizada inicialmente en la sala de angiografía digital gracias a la ayuda prestada por los radiólogos José Antonio Recondo y Mariano de Blas. La gran personalidad y profesionalidad de José Ramón Ribera, Jefe del Servicio de Cirugía, hacía que su Servicio fuera probablemente insignia del Hospital, con capacidad Docente, muchas veces recurríamos a sus Residentes para que nos echaran una mano en algunas intervenciones quirúrgicas y en muchas urgencias. Teníamos mucha relación con el Servicio de Traumatología, tanto con su Jefe de Servicio (Juan Ignacio Querejeta) como con el resto del equipo, fundamentalmente al incorporarse Enrique Moreno y Antonio Martínez, con quienes su afición por la columna hizo que colaboráramos en algunos casos, fundamentalmente de instrumentación vertebral. Las sesiones clínicas que realizábamos en la máquina de café o en la consulta de mi amigo Julio Igartua Sagarminaga (Jefe de ORL) con el café bien preparado por nuestra Arantxa Zapirain, eran bien conocidas y asistíamos asiduamente Ernesto Casis, Iñaki Hernaez y yo junto a otros esporádicos colegas cirujanos, urólogos, rehabilitadores, traumatólogos... hacíamos auténticas reuniones interdisciplinarias, allí resolvíamos casos

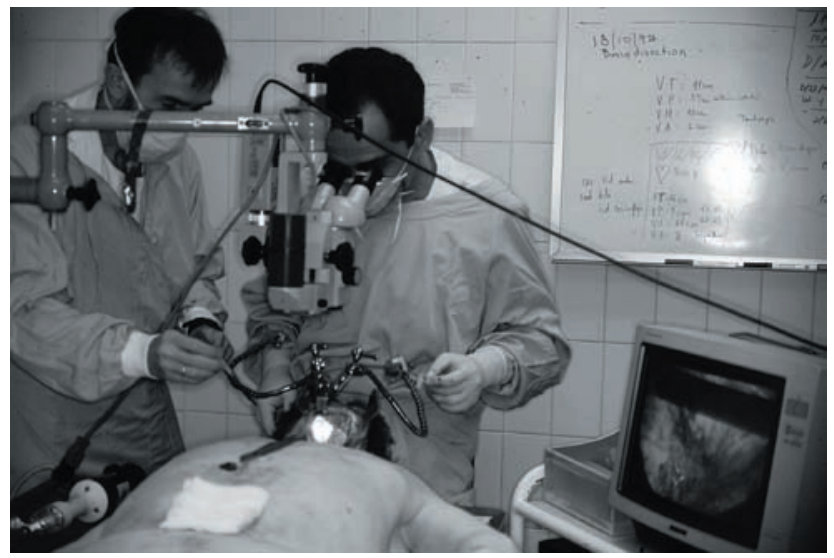

Figura 14. El Dr. Juan Proaño y a su izquierda el que suscribe, en la sala de autopsias del Servicio de Anatomía Patológica del Hospital Provincial de Guipúzcoa 1994.

difíciles, pero también se hablaba de todo: de lo divino y de lo humano, llegando con los años a bautizar las charlas de café como "la bodeguilla". Se celebraban Juntas Facultativas donde también participábamos los responsables de las Secciones a la par que las jefaturas de Servicios. Eran otros tiempos. También hacíamos entrenamiento en microcirugía e investigación, ya que nuestro Hospital contaba con un pequeño animalario de donde nos surtíamos para practicar microcirugía en una sala experimental localizada en lo que llamábamos "el submarino" (Fig.13). Incorporamos un instrumental básico desechado del quirófano así como el viejo microscopio quirúrgico a la sala de autopsias del Servicio de Anatomía Patológica (Fig.14), cuyo excelente Jefe de Servicio D. Antonio Gastaminza, nos permitía realizar estudios anatómicos que muchos de ellos han sido publicados en nuestra revista "Neurocirugía"19-21.

Finalmente tuvimos que rendirnos a los tiempos modernos, aprendiendo términos de gestión hospitalaria como eficacia, eficiencia, planificación, equidad, calidad... y con ello aceptar que la coordinación primero y luego la unificación de Servicios y Hospitales era inevitable, dando paso a otra estructura de mayor complejidad que hoy recibe el nombre de Hospital Donostia. En fin, de esta forma, en Mayo del 2000 fuimos junto con cirugía vascular de las primeras unidades clínicas fusionadas dentro del llamado Complejo Hospitalario Donostia, pero esta ya es otra historia.

\section{Agradecimientos}

Quiero expresar mi mas profundo agradecimiento por su inestimable ayuda y aportación de datos con rigor histórico sin los cuales no se hubiera podido realizar este trabajo a los Doctores: Mariano Arrazola, Fernando Asuero, Jesus María Bidaguren, Antonio Díaz Aramendi, Miguel Eche- 
nique, José Luis Elorza, Carlos Elósegui, Luis Guereca, Julio Igartua, José María Izquierdo, José Luis Munoa, Javier Olascoaga, Antonio Palacios, José Ramón Ribera, Javier Urcola, Ramón Urquizu, José María Urkia, Gabriel Zubillaga y José María Zapirain. Al Sr. D. Baltasar Marín (primer Director Gerente del Hospital Provincial (19801988). Asimismo agradecer a los fotógrafos del Hospital de Guipuzcoa D. Antonio González Lemos y D. Javier Alonso Martín, asi como a la Unidad de Comunicación del Hospital Donostia por accederme a bucear en el archivo fotográfico del Hospital. A Marta de Miguel, mi mujer, sin cuyo apoyo, comprensión y paciencia no hubiera podido estar donde estoy ni realizar esta historia.

\section{Bibliografía}

1. Albert, P.: Desarrollo y planificación de las unidades asistenciales y docentes en Neurocirugía. Neurocirugía 1993; 4: 172-176.

2. Arrazola Silio, M., Arrazola Schlamilch M., Urculo Bareño, E.: Observations regarding 2.550 patients operated upon for protuded lumbar intervertebral disc. Book of Abstracts. $8^{\text {th }}$. European Congress of Neurosurgery. Barcelona. Spain. 1987; pp. 234.

3. Arrazola Silio, M., Arrazola Schlamilch M., Urculo Bareño, E.: Delayed epidural hematoma. Book of Abstracts. $9^{\text {th. }}$ International Congress of Neurological Surgery. New Delhi-India. 1989; pp. 78.

4. Boixadós, J.R., Arrazola, M.: Malformaciones de la región occipito-cervical. En Obrador, S., Albert, P., Anastasio, J.V., Arrazola, M., Boixadós, J.R., Sánchez Juan, J., Vázquez Añón, J.J. (ed). Síndromes neurológicos en las malformaciones y lesiones degenerativas del estuche cráneo-vertebral y su tratamiento neuroquirúrgico. Instituto de investigaciones clínicas y médicas. Madrid. Monografía núm 2. Paz Montalvo, 1956; pp. 99-198.

5. Bravo, G.: Perspectives in International Neurosurgery: Neurosurgery in Spain. Neurosurgery 1984; 14: 111-112.

6. Gorritxo, J.A.: Prólogo. En: Estudio sobre el complejo hospitalario de Zorroaga. Departamento de sanidad y seguridad social del gobierno vasco. Gráficas Santamaría S.A. Vitoria-Gasteiz 1983.

7. Greenblat, S.H., Dagi, T.F., Epstein, M.H.: A History of Neurosurgery. In its scientific and professional contexts. The American Association of Neurological Surgeons. Park Ridge, Illinois. 1997.

8. Gutiérrez, D., Izquierdo, J.M.: El doctor Obrador en la Medicina de su tiempo. Oviedo. 1984.

9. Izquierdo, J.M.: Historia de la Neurocirugía española (1950). Neurocirugía 1993; 4: 164-171.

10. Izquierdo, J.M.: Concepto y desarrollo de la Neurocirugía. En Izquierdo, J.M., Blázquez, M.G., Coca,
2009 20: 163-175

J.M., Argüello, C. Fundamentos de Neurocirugía. Gráficas Summa, S.A. Oviedo. 1979; pp.1-33.

11. Izquierdo, J.M.: Diario de un residente. Septem Ediciones, S.L., Oviedo, 2004.

12. Izquierdo, J.M., Martín, R., Pinto, J.I.: Neurocirugía básica para residentes. Sociedad Española de Neurocirugía. 2007.

13. Lamas, E.: Influencia de la personalidad de Sixto Obrador en su obra médica. Neurocirugía 1999; 10: 417-419.

14. Martín-Rodríguez, J.G.: Color still and motion photography and color televisión recording through the operating microscope. En Rand, R.W. (ed). Microneurosurgery. The C.V. Mosby Company, 1985; pp. 83-91.

15. Martínez Lage, J.M., López, S., Tolosa, E.: Autobiografía de la Sociedad Española de Neurología (1949-1994) y otras Memorias de la Neurología Española. Fundación Uriach, 1995; pp: 303-333.

16. Obrador, S., Fernández Urdanibia, J., Soto Cuenca, M., Martín Rodríguez, J.G.: Observaciones en una serie de cien enfermos neuroquirúrgicos estudiados con tomografía axial computarizada. Neurocirugía Luso-Española. 1976; 16: 9-25.

17. Poza, M.: Historia de la SLEN. Reflejos de las actas. Neurocirugía 1993; 4: 92-94.

18. Úrculo Bareño, E., Onzain Behobide I.: Meningiomas múltiples. Terminología, diagnóstico y tratamiento. Rev. Esp. Neurol. 1989; 4: 557-561.

19. Úrculo, E., Martínez, L., Arrazola, M., Aranzadi, M..J., Gastaminza, A.: Estudio anatomorradiológico de la técnica de Mullan en el tratamiento de la neuralgia del trigémino. Neurocirugía 1994; 5: 107-114.

20. Úrculo Bareño, E.: Anatomía quirúrgica de la porción cisternal del IV par craneal. Neurocirugía 1997; 8: 163-170.

21. Úrculo, E., Arrazola, M.: Estudio anatomorradiológico de la microcompresión percutánea del nervio trigémino. Neurocirugía 1998; 9: 61.

22. Úrculo, E., Arrazola, M., Gereka, L., Olasagasti, V., Olascoaga, J., Urcola, J., Zabalza, R.: Valoración de la técnica de Mullan en el tratamiento de la neuralgia del trigémino. Rev. Neurol. 1998; 27 (157): 477-484.

23. Urkia, J.M.: Cien años de Medicina en Gipuzkoa 18991999. Fundación Kutxa. 1999.

24. Wertheimer, P., David, M., Sindou, M., Redondo, A.: Naissance et croissance de la neurochirurgie. Neuro-Chirurgie 1979; 25: 247-363.

Úrculo Bareño, E.: Historia de la Neurocirugía en el Hospital Provincial de Guipúzcoa. Neurocirugía 2009; 20: 163-175.

Correspondencia postal: Enrique Úrculo. Servicio de Neurocirugía. Hospital Donostia. Paseo del Doctor Beguiristain s/n. San Sebastian. 